\title{
SAÚdE MENTAL DE PSICÓlOgOS ATUANTES EM SERVIÇOS DE ATENÇÃO PRIMÁRIA À SAÚDE
}

\author{
Lucas Có Barros Duarte \\ Universidade Federal do Espírito Santo \\ Thiago Drumond Moraes \\ Universidade Federal do Espírito Santo
}

\begin{abstract}
Resumo
Este estudo objetivou conhecer e caracterizar o trabalho do psicólogo na atenção primária à saúde e seus efeitos na saúde desses profissionais. Participaram da pesquisa 32 psicólogos que atuam em serviços públicos de atenção à saúde no estado do Espírito Santo. Os participantes responderam a um questionário online composto pelos seguintes instrumentos: JCQ, WHOQOL-bref, SRQ-20; e questões sociodemográficas. Os resultados indicam que o trabalho do psicólogo nesses espaços caracteriza-se enquanto trabalho ativo, no entanto observou-se um nível elevado de adoecimento mental e baixa qualidade de vida dentre os participantes. Além disso destacou-se, na saúde mental dos trabalhadores, uma maior influência da demanda psicológica do trabalho do que do controle. A dimensão controle parece não contribuir positivamente para a saúde dos trabalhadores como previsto no modelo teórico. Por fim, indicam-se estratégias para melhoria do trabalho, com foco na demanda psicológica.
\end{abstract}

Palavras-chave: saúde ocupacional; condições de trabalho; atuação do psicólogo; serviços de saúde pública.

\section{MENTAL HEALTH OF PSYCHOLOGISTS WORKING IN PRIMARY HEALTH CARE SERVICES}

\begin{abstract}
This study aimed to characterize the work of psychologists in primary health care and its effects on their health. The participants were 32 psychologists working in public health care services in the state of Espírito Santo. Participants responded to an online questionnaire consisting of the following instruments: JCQ, WHOQOL-BREF, SRQ-20; and sociodemographic questions. The results indicate that the psychologist's work in these areas is characterized as active work, however there was a high level of mental illness and poor quality of life among the participants. Also highlighted is a greater influence of psychological demands than the influence of control on workers' mental health. The dimension "control" does not seem to contribute positively to the health of workers as hypothesized by the theoretical model. Finally, strategies for improving these professionals' work are indicated, focusing on the psychological demand.

Keywords: occupational health; working conditions; psychologist performance; public health services.
\end{abstract}




\title{
SALUD MENTAL DE PSICÓlOgOS EN SERVICIOS DE ATENCIÓN PRIMARIA DE SALUD
}

\begin{abstract}
Resumen
Este estudio tuvo como objetivo caracterizar el trabajo del psicólogo en la atención primaria de salud y sus efectos sobre la salud de estos profesionales. Los participantes fueron 32 psicólogos que trabajaban en servicios de salud pública del estado de Espírito Santo; los cuales respondieron un cuestionario en línea compuesto por los instrumentos: JCQ, WHOQOL-BREF, SRQ-20 y preguntas sociodemográficas. Los resultados indican que el trabajo del psicólogo en estos espacios se caracteriza por ser un trabajo activo, pero se observó un nivel elevado de enfermedad mental y baja calidad de vida entre los participantes. Se destacó en la salud mental de los trabajadores, una mayor influencia de la demanda psicológica del trabajo más que del control. La dimensión control no pareció contribuir positivamente a la salud de los trabajadores como es previsto en el modelo teórico. Por último, se indican estrategias para mejorar el trabajo enfocadas en la demanda psicológica.

Palabras clave: salud ocupacional; condiciones de trabajo; actuación del psicólogo; servicios de salud pública.
\end{abstract}

\section{INTRODUÇÃO}

Devido às diversas transformações ocorridas nos modos de produção e na organização do trabalho ao longo dos últimos séculos - as revoluções industriais, a organização científica do trabalho, automatização e avanços tecnológicos, instabilidade no emprego, aumento da produtividade, etc. - o tema saúde mental do trabalho tem se tornado cada vez mais presente nos ambientes de trabalho e nas produções científicas (Borsoi, 2007; Doppler, 2007; Knuth et al., 2015). No entanto, é possível observar que em muitos dos estudos e pesquisas desenvolvidas a respeito dessa temática, estabelece-se uma relação direta entre o trabalho do modo como está organizado atualmente e o adoecimento, ou seja, enfatizando os aspectos negativos do trabalho e, muitas das vezes, negligenciando os aspectos positivos que o trabalho possui na vida das pessoas (Doppler, 2007; Guérin, Laville, Daniellou, Duraffourg \& Kerguelen, 2001).

Guérin et al (2001) afirmam que as relações entre o trabalho e a saúde do trabalhador se dão de modos complexos, sendo que o trabalho pode desempenhar um papel positivo ou negativo na saúde do trabalhador de acordo com as condições encontradas para realiza-lo. Guérin et al (2001) sugerem ainda que as "agressões à saúde", papel negativo do trabalho na saúde, podem ser a curto ou a longo prazo, observáveis ou não, e físicas, como tradicionalmente discutido pela medicina do trabalho, ou psíquicas, como mais recentemente demonstrado pela psicopatologia do trabalho.

Várias áreas e teorias se propuseram a estudar os processos saúde-doença associados ao ambiente de trabalho, como: a medicina do trabalho, as teorias do estresse, a psicopatologia do trabalho, a psicodinâmica do trabalho, saúde e segurança do trabalho, ergologia e ergonomia. Algumas delas enfocando ora aspectos físicos, psíquicos, individuais, coletivos ou ambientas, ou, ainda, buscando englobar vários desses aspectos. Jacques (2003) destaca quatro amplas abordagens que se propõem a estudar as relações entre trabalho e 
processos de saúde-doença mental, sendo elas: "as teorias sobre estresse, a psicodinâmica do trabalho, as abordagens de base epidemiológica e/ou diagnóstica e os estudos e pesquisa em subjetividade e trabalho" (p.100). Este estudo pretende articular o modelo de estresse de Karasek (1979) - Demanda e Controle - com instrumentos de mensuração da saúde mental (SRQ-20) e qualidade de vida (Whoqol-bref), tendo ainda como referência a perspectiva sócio histórica do trabalho.

\section{Saúde Mental e Trabalho}

Desde o início do século XX já haviam estudos da American Journal of Psychiatry voltados para a saúde mental ocupacional, psiquiatria ocupacional e psiquiatria industrial (Seligmann-Silva, 1994). No entanto, a expressão "Psicopatologia do Trabalho" surgiu apenas em 1952, na França, em um artigo de Paul Sivadon. Este autor, respaldado por experiências em hospitais psiquiátricos no período pós II Guerra Mundial, "estabeleceu uma nova forma de abordar o doente mental e permitiu reconhecer o trabalho pelo seu valor de integração social, oferecendo-nos grande contribuição para os estudos na área de saúde mental no trabalho" (Nassif, 2005, p. 81), a Ergoterapia. Conforme Nassif, a Ergoterapia é um método psicoterápico voltado para a cura das doenças por meio do trabalho. A partir desses trabalhos, Sivadon lança luz sobre o fator social do trabalho e seu papel positivo para a saúde do indivíduo, ampliando a visão anterior do trabalho enquanto causador de doenças.

Posteriormente, Le Guillant, baseando-se nos trabalhos de Follin e Bonnafé e de Georges Politzer, desenvolveu várias pesquisas no campo da psicopatologia do trabalho, com um enfoque psicossociológico da doença mental, no qual o trabalho figura como instância central da realidade social (Nassif, 2005). No entanto, mesmo com as significativas contribuições de Le Guillant para a área, esse autor ressaltava a dificuldade de determinar uma conexão entre os distúrbios e as atividades de trabalho (Nassif, 2005).

Diversos estudos (Borges \& Argolo, 2002; Elias \& Navarro, 2006; Martinez, 2002; Martinez, Paraguay \& Latorre, 2004; Nascimento Sobrinho, Carvalho, Bonfim, Cirino \& Ferreira, 2006; Rocha \& Fernandes, 2008) têm buscado verificar e compreender essas possíveis relações entre saúde mental e trabalho. Para isso, muitas das vezes utilizam de instrumentos diagnósticos e/ou de avaliação, como escalas de estresse, qualidade de vida e burn-out, ou instrumentos de triagem como o SRQ-20 (Araújo, Aquino, Menezes, Santos \& Aguiar, 2003; Araújo, Sena, Viana \& Araújo, 2005; Cardoso, Ribeiro, Araújo, Carvalho \& Reis, 2009; De Marco, Cítero, Moraes \& Nogueira-Martins, 2008; Kirchhof et al, 2009;). Dessa forma, eles tentam relacionar psicopatologias e processos de saúde-doença mental a características do mundo do trabalho, como condições de trabalho, carga horária, carga de trabalho, etc. 
Com outra forma de abordar a temática saúde mental no trabalho, desenvolveram-se no final do século $X X$ as chamadas teorias do estresse. 0 conceito de estresse tem sua origem na física e posteriormente foi utilizado pelo médico e fisiologista austríaco Hans Seyle, em 1936, para denominar a "síndrome geral de adaptação", um processo de dimensão nitidamente biológica, onde o organismo responde às ameaças do ambiente, sendo constituído por três fases: reação de alarme, fase de adaptação e fase de exaustão (Borsoi, 2007; Filgueiras \& Hilppert, 2002; Jacques, 2003).

A psicologia se apropriou deste conceito, ampliando o entendimento para além da dimensão biológica, quando Lazarus e Folkman (citados por Jacques, 2003) definiram estresse psicológico "como uma relação entre a pessoa e o ambiente que é avaliado como prejudicial ao seu bem-estar" (p. 101). Jacques aponta a ênfase desses autores para a avaliação cognitiva que a pessoa faz da situação estressora, o que permite esforços de enfrentamento (coping) dela.

\section{Modelo Demanda e Controle}

Com relação aos modelos psicológicos de estresse, Falzon e Sauvagnac (2007) apresentam dois modelos principais, sendo os transacionais e os interacionistas; o autor associa ainda a esses modelos os trabalhos sobre burnout. De acordo com os autores, os modelos transacionais focalizam-se nos comportamentos dos indivíduos, analisando pouco o contexto de trabalho e o ambiente. Esses modelos propõem a gestão do estresse, por meio de intervenções no indivíduo, como maneira de solucionar os problemas do estresse psicológico no trabalho. Os modelos interacionistas integram uma avaliação do ambiente, que pode ser considerado patogênico em maior ou menor grau, tendo efeito diferenciado nos indivíduos, a depender do seu grau de sujeição ao estresse. Dentre os modelos interacionistas, Falzon e Sauvagnac destacam o modelo de Karasek e o modelo de Siegrist, afirmando ainda que o modelo de Karasek seja, provavelmente, o melhor avaliado e com maior repercussão internacional.

O Modelo Demanda-Controle foi elaborado por Karasek (1979) a partir de críticas aos modelos unidimensionais que abordavam o estresse ocupacional, sendo largamente utilizado em países da Europa, na América do Norte e no Japão (Araújo, Graça \& Araújo, 2003). Nele são tratadas duas dimensões referentes ao ambiente de trabalho: as demandas psicológicas advindas do trabalho e o controle do trabalhador sobre seu trabalho. As demandas psicológicas se referem a exigências psicológicas enfrentadas pelo trabalhador para realizar suas tarefas, "envolvendo pressão do tempo (proporção do tempo de trabalho realizado sob tal pressão), nível de concentração requerida, interrupção das tarefas e necessidade de se esperar pelas atividades realizadas por outros trabalhadores" (Araújo, Graça et al, 2003, p. 993). 
O controle do trabalhador sobre suas atividades é composto por dois fatores. O primeiro refere-se aos aspectos do uso de habilidades, ou seja, o grau tido pelo trabalho ao envolver aprendizagem, desenvolvimento de habilidades, tarefas variadas, repetitividade e criatividade. O segundo é denominado autoridade decisória, que "abarca a habilidade individual para a tomada de decisões sobre o próprio trabalho, a influência do grupo de trabalho e a influência na política gerencial" (Araújo, Graça et al., 2003, p. 993).

A partir dessas dimensões o modelo analisa os riscos à saúde, provenientes de situações no ambiente laboral. Karasek (1985) criou um instrumento para avaliar essas dimensões, chamado Job Content Questionnaire (JCQ), compreendendo 49 questões que tratam de demanda (física e psicológica), controle, suporte social, e insegurança no emprego. Esse instrumento foi validado a partir dos estudos com amostras de trabalhadores representativas nos Estados Unidos e na Suécia (Araújo, Graça et al., 2003).

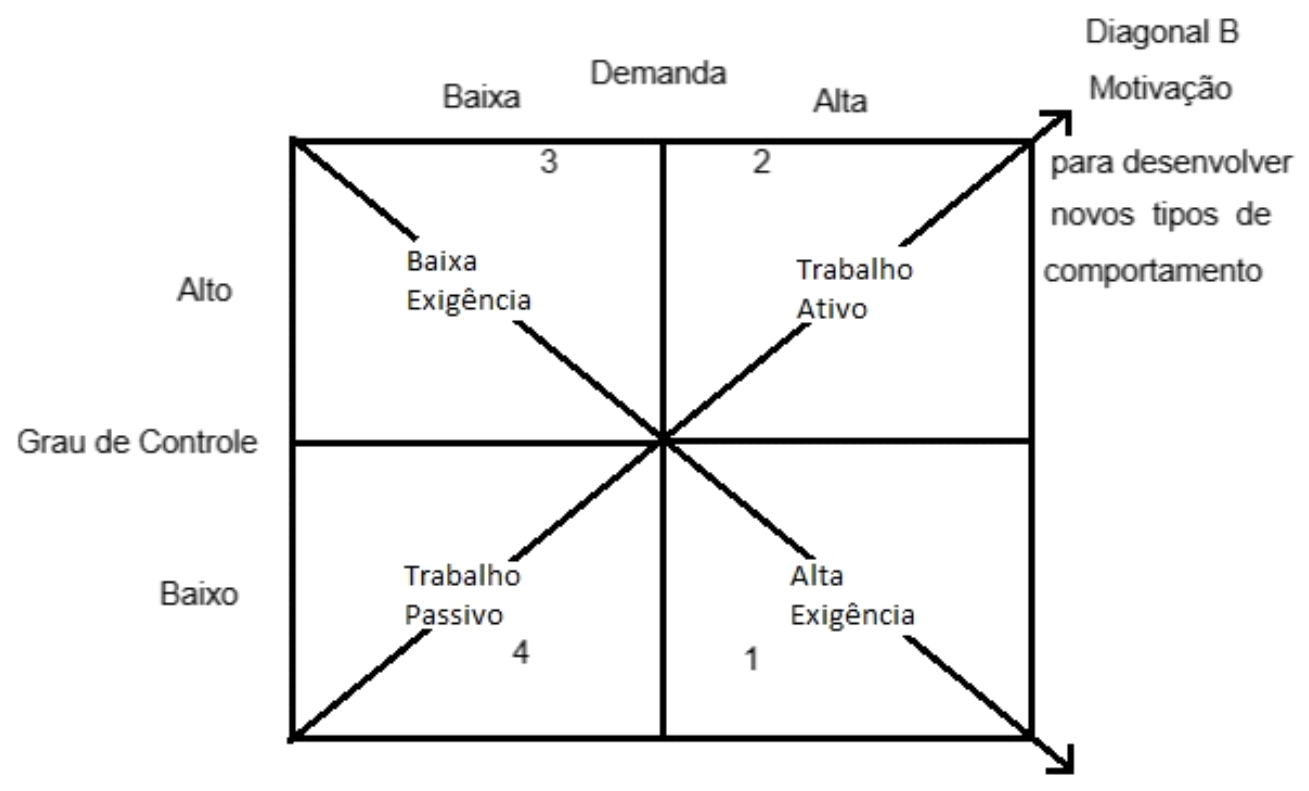

Diagonal A

Riscos de exigência

psicológica e adoecimento psíquico

Figura 1. Modelo Demanda-controle de Karasek (Fonte: Adaptado de Karasek, 1979).

Como se pode observar na Figura 1, esse modelo identifica quatro tipos de experiências de trabalho, de acordo com os níveis de demanda psicológica e de controle que o trabalhador vivencia, sendo: "trabalho passivo" (baixa demanda baixo controle), "trabalho ativo" (alta demanda - alto controle), "alta exigência do trabalho" (alta demanda - baixo controle) e "baixa exigência" (baixa demanda - alto controle) (Araújo, Graça et al., 2003; Karasek, 1979; Santos, 2006). As 
diagonais $A$ e $B$, apresentadas na Figura 1 referem-se, respectivamente, ao risco de adoecimento psíquico e físico, e à motivação para desenvolver novos modos de comportamento. Assim o quadrante 1 (alta exigência) representa a situação de trabalho com a maior tendência a "reações adversas das exigências psicológicas, tais como fadiga, ansiedade, depressão e doença física" (Araújo, Graça et al., 2003, p. 993). No entanto, a situação representada no quadrante 4 (trabalho passivo), "pode conduzir ao declínio na atividade global do indivíduo e à redução da capacidade de produzir soluções para as atividades e problemas enfrentados" (Araújo, Graça et al., 2003, p. 993).

Um estudo de revisão bibliográfica realizado por Greco, Magnago, Prochnow, Beck e Tavares (2011) traz o panorama do uso do JCQ na América Latina. As autoras encontraram, a partir desse levantamento, 35 publicações, sendo 30 artigos e cinco teses produzidas no Brasil. Destaca-se o número de trabalhos realizados com profissionais da saúde, mas apenas médicos e enfermeiros, não tendo sido encontrado nenhum trabalho com profissionais de outras categorias. Em 2013, foi produzida uma dissertação de mestrado que visou investigar os efeitos indiretos de uma capacitação com profissionais de psicologia atuantes em serviços públicos para o atendimento de pessoas vítimas de violência sexual (Freitas, 2013); para atingir tal objetivo a autora utilizou o JCQ juntamente com o Maslach Burnout Inventory (MBI). Até o momento, esse trabalho foi o único publicado utilizando o JCQ em uma população de psicólogos.

\section{Qualidade de Vida no Trabalho}

Apesar de ser um conceito amplamente utilizado e discutido, pode-se perceber que não há um consenso a respeito do que é qualidade de vida. Minayo (2000), diz que "a noção de qualidade de vida transita em um campo semântico polissêmico" (p.10), pois alguns autores buscam trabalha-la de forma universal, baseando-se na concepção de que há elementos relativos à qualidade de vida minimamente comuns nas diversas culturas; enquanto outros autores destacam a faceta contextual desse conceito, localizando as noções de qualidade de vida em recortes temporais, espaciais, sociais, etc. Fleck et al. (1999), apontam que o termo "qualidade de vida", foi utilizado pela primeira vez em 1964, pelo então presidente dos Estados Unidos, Lyndon Johnson, quando declarou que "os objetivos não podem ser medidos através do balanço dos bancos. Eles só podem ser medidos através da qualidade de vida que proporcionam às pessoas" (p. 20).

Para alguns autores, este conceito é entendido como "a percepção do indivíduo de sua posição na vida no contexto da cultura e sistema de valores nos quais ele vive e em relação aos seus objetivos, expectativas, padrões e preocupações" (WHOQOL Group, 1994, citado por Fleck et al, 1999). Assim, reconhece-se que a qualidade de vida é um construto multidimensional, abrangendo aspectos individuais e socioculturais da vida dos indivíduos. 
Minayo (2000) destaca que a qualidade de vida se refere à satisfação de necessidades primárias para a vida, pautadas em "noções relativas de conforto, bem-estar e realização individual e coletiva" (p. 10), como saúde, alimentação, saneamento básico, moradia, educação, transporte, saúde, lazer, trabalho. Partindo-se desse entendimento sobre qualidade de vida, será ressaltada, neste estudo, a importância que o trabalho desempenha para as pessoas da cultura ocidental.

Assim como o conceito de qualidade de vida, a Qualidade de Vida no Trabalho (QVT) também apresenta uma série de definições e noções divergentes entre os estudiosos, ao longo do tempo (Lacaz, 2000). A partir do proposto pelo Programa Internacional para o Melhoramento das Condições e dos Ambientes de Trabalho (PIACT), da Organização Internacional do Trabalho (OIT), em 1976, o entendimento que se tem sobre a QVT tenta articular duas tendências: a primeira compreendendo a QVT como parte da noção de qualidade geral de vida; a outra, como algo voltado para o aumento da participação dos trabalhadores no ambiente de trabalho e na sua vida profissional (Lacaz, 2000; Mendes, 1988).

Nesse artigo optou-se pelo uso da noção de qualidade de vida, por entender-se que essa engloba também o conceito de qualidade de vida no trabalho, como mostra a definição supracitada da OIT. Ademais, pretendeu-se utilizar, numa perspectiva mais ampla, a qualidade de vida enquanto um dos fatores relacionados à saúde dos trabalhadores, não se restringindo ao ambiente de trabalho e/ou vida profissional; partindo do pressuposto da perspectiva sócio histórica do trabalho (Clot, 2006, 2010) de que a vida profissional não se desvincula de outros aspectos da vida dos trabalhadores.

\section{Saúde do psicólogo}

Medeiros, Nunes e Melo (2012) afirmam que há poucas pesquisas realizadas sobre a saúde do psicólogo e, em consulta à literatura, percebe-se que os estudos sobre o tema são comumente realizados com profissionais que atuam na área hospitalar.

Silva (2010), em uma revisão de literatura sobre a saúde mental do psicólogo atuante em Unidades de Terapia Intensiva (UTI), relatou algumas questões prejudiciais à saúde desses profissionais, como a sobrecarga da jornada de trabalho, a falta de clareza no desempenho de sua função, o não reconhecimento por parte dos colegas a respeito da atividade que desenvolve, despreparo emocional para lidar com as situações que passam no cotidiano do hospital, entre outros. Além disso, Medeiros et al. (2012) destacam que há uma maior preocupação acadêmica em produzir estudos voltados para a melhoria das condições psíquicas dos pacientes, e se negligencia a saúde do trabalhador e sua necessidade de cuidado.

Em estudo realizado com profissionais da Atenção Primária à Saúde (APS), Brotto e Dalbello-Araujo (2012) questionam a inerência do adoecimento no 
trabalho em saúde, concluindo que a saúde desses profissionais é prejudicada, sobretudo, pelos seus processos de trabalho. O conceito de processo de trabalho utilizado é baseado nos escritos de Karl Marx (2008), sendo entendido como um conjunto de atividades planejadas com a finalidade de fabricação de valores de uso, apropriando-se de matérias da Natureza para satisfazer as necessidades humanas. Marx (2008) afirma ainda que o processo de trabalho se finda no produto. Sanna (2007) destaca seis componentes do processo de trabalho, a saber: o objeto, os agentes, os instrumentos, as finalidades, os métodos e, por fim, os produtos.

Com relação à atuação da psicologia, destaca-se que os profissionais inseridos em serviços de saúde ficam de certa forma desorientados, desenvolvendo muitas vezes a prática clínica e não uma prática hospitalar (Medeiros et al., 2012). Ademais é relatada a dificuldade dos psicólogos em atuar juntamente com a equipe, desenvolvendo uma atividade solitária e pouco reconhecida pelos colegas. Essa falta de clareza e orientação para o desempenho da prática do psicólogo na saúde também é percebida nos outros níveis de atenção, como nos espaços das Unidades Básicas de Saúde, sendo, frequentemente, reproduzido o modelo clínico, reconhecidamente clássico, na atuação desses profissionais (Archanjo \& Schraiber, 2012), que muitas vezes leva a uma sobrecarga de trabalho.

Muitos autores nos chamam a atenção para uma questão que pode comprometer a atuação do psicólogo na APS (Boarini \& Borges, 2009; Dimenstein, 1998; Romagnoli, 2009), enfatizando a própria formação desse profissional. Devido a uma cultura de atuação estabelecida na regulamentação da profissão (Dimenstein, 1998), há "uma tendência hegemônica de atuação do psicólogo, centrado no caráter privatista e individualizante de atendimento" (Boarini \& Borges, 2009, p.604). Assim, um longo debate tem se dado no que concerne às diretrizes curriculares da graduação em Psicologia, de modo a garantir novas formas de atuação desse profissional. Busca-se, com isso, fazer com que o psicólogo atue de forma mais eficaz junto aos demais profissionais das equipes de saúde. Romagnoli afirma que "embora uma parcela significativa de psicólogos trabalhe na área da saúde, ainda há um hiato entre a formação encontrada nos cursos de Psicologia e as demandas dessa inserção" (2009, p.527).

Considerando a importância crescente do psicólogo nas equipes de saúde, é relevante estudar os fatores que levam esses profissionais ao adoecimento, a fim de pensar em estratégias que melhorem sua saúde e atuação. Partindo da hipótese de que os psicólogos atuantes em serviços de atenção primária têm adoecido por motivos de trabalho, essa pesquisa teve o objetivo de conhecer e caracterizar o trabalho do psicólogo na atenção primária e seus efeitos na saúde desses profissionais. Para isso foram utilizados o instrumento proposto pelo 
modelo demanda-controle, e instrumentos de mensuração de qualidade de vida e saúde mental.

\section{MÉTODO}

Para a realização desta pesquisa foi adotada uma abordagem quantitativa do objeto a ser estudado, a fim de se conhecer e verificar algumas características do trabalho de psicólogos atuantes em serviços de atenção à saúde, bem como os impactos dessa atuação na saúde mental desses trabalhadores.

\section{Participantes}

Participaram desse estudo psicólogos que atuam em serviços públicos de atenção à saúde no estado do Espírito Santo, sendo um total de 32 participantes. Vale ressaltar que não há um quantitativo exato da população investigada, estimando-se que haja aproximadamente 300 psicólogos atuando serviços de Atenção Primária à Saúde no estado do Espírito Santo, de acordo com o CRP-16 e o Sindicato de Psicólogos do Espírito Santo (Sindpsi-ES). Essa estimativa pode ser feita com base no número de municípios do Estado, considerando que na maioria dos municípios há pelo menos um psicólogo atuando na saúde. Apesar de boa parte das prefeituras possuírem o chamado "Portal da Transparência", a informação do número de profissionais atuando por cargo e/ou lotação não é obtida de maneira clara e rápida, sendo que muitas vezes inexiste esse tipo de dados.

Dentre os respondentes, 29 disseram atuar em serviços de Atenção Primária, enquanto três trabalham em serviços de média complexidade - por exemplo, CAPS. A idade dos participantes variou de 24 a 60 anos ( $M=35,03$; $\mathrm{DP}=9,15)$, sendo 27 do sexo feminino. Demais informações a respeito da população estão apresentadas na Tabela 1 e na Tabela 2.

\section{Instrumentos}

Foi solicitado aos participantes o preenchimento de um questionário online, contendo os seguintes instrumentos:

\section{WHOQOL-bref}

Desenvolvido pelo Grupo de Qualidade de Vida da Organização Mundial de Saúde (WHOQOL Group), e validado em português por Fleck et al. (2000). Este instrumento avalia qualidade de vida e contém 26 questões divididas em quatro domínios: físico, psicológico, relações sociais e meio ambiente. As questões são respondidas de acordo com uma escala tipo Likert, variando entre 1 e 5, sendo que, entre as 26 questões, há quatro tipos de escalas de resposta: intensidade, capacidade, avaliação e frequência. 
Self-Reporting Questionnaire (SRQ-20)

O SRQ-20 é a versão de 20 itens do SRQ-30 para rastreamento de transtornos mentais não-psicóticos. Foi validado primeiramente no Brasil por Mari e Williams $(1985,1986)$ e mais recentemente revalidado por Gonçalves, Stein e Kapczinski (2008). As questões são respondidas em sim ou não, e as respostas afirmativas pontuam 1 no somatório final dos valores. O escore final indica a probabilidade de presença de transtorno não-psicótico, variando de 0 (nenhuma probabilidade) a 20 (extrema probabilidade).

Job Content Questionnaire (JCQ)

Criado por Karasek (1985) para medir aspectos psicossociais do trabalho. Foi validado em português por Araújo e Karasek (2008) e chamado de Questionário sobre o Conteúdo do Trabalho. Sua versão mais recente contém 49 questões divididas nas seguintes escalas: a) Controle sobre o trabalho incluindo uso de habilidades (6 questões), autoridade decisória (3 questões) e autoridade decisória no nível macro (8 questões); b) Demanda psicológica - 9 questões; c) Demanda física - 5 questões e d) Suporte social - 11 questões (5 sobre suporte social proveniente da chefia e 6 de suporte proporcionado pelos colegas de trabalho); e) Insegurança no trabalho - 6 questões; e f) Uma questão sobre nível de qualificação exigida para o trabalho que é executado (corresponde ao nível educacional que é requerido no posto de trabalho ocupado).

\section{Caracterização demográfica}

Além desses instrumentos, o questionário continha uma página com perguntas de cunho sociodemográfico (por exemplo, idade, sexo, formação) e com questões a respeito de algumas características relacionadas ao trabalho do participante (carga horária, tempo que atua no serviço, remuneração, etc.).

\section{Procedimentos}

O projeto foi aprovado pelo Comitê de Ética em Pesquisa da Universidade Federal do Espírito Santo, sob o número do parecer 1.065.962, no dia 08 de maio de 2015.

Primeiramente entrou-se em contato com o Conselho Regional de Psicologia do Espírito Santo (CRP-16), a fim de se estabelecer uma parceria que viabilizasse a coleta online dos dados. Após a consolidação dessa parceria, foi confeccionado o questionário na página do Google Forms para dar início à aplicação online. O questionário foi enviado por quatro vezes, através da lista de e-mails do CRP-16, a todos os psicólogos registrados no estado do ES. Além da divulgação do questionário pelo CRP-16, também se optou por divulga-lo em páginas e grupos de redes sociais.

Os questionários continham uma página inicial com esclarecimentos a respeito da pesquisa, no qual era apresentado o caráter voluntário da pesquisa, 
assim como informações acerca da confidencialidade e anonimato dos dados, e contatos dos pesquisadores responsáveis. O questionário permaneceu online por um período de três meses, entre julho e outubro de 2015, tendo sido desconsiderada respostas após essa data.

Os dados obtidos por meio da coleta foram analisados através do software IBM-SPSS v.20. Primeiramente buscou-se descrever a população por meio dos dados sociodemográficos. Em seguida, realizou-se a construção dos indicadores das escalas. Para o JCQ, seguiu-se as orientações do Job Content Questionnaire User's Guide (Karasek, 1985). Utilizando-se das fórmulas trazidas no manual, calculou-se os indicadores de demanda, de apoio social e de controle, fazendo-se o somatório das variáveis que constituem cada um desses indicadores. Por fim, realizou-se a dicotomização desses indicadores entre alto e baixo, estabelecendo-se como ponto de corte a mediana referente a cada indicador.

Para o SRQ-20, fez-se o cálculo dos escores obtidos pelos participantes no instrumento, considerando respostas positivas tendo valor um, e respostas negativas com valor zero. A partir desses escores, criou-se uma variável categórica dicotômica (" $d p m^{\prime \prime)}$, caracterizando o participante como provável portador de distúrbios psíquicos menores, ou não portador, utilizando-se como ponto de corte os valores $7 / 8$, de acordo com a avaliação realizada por Gonçalves et al. (2008). Com relação ao WHOQOL-bref, fez-se o cálculo dos quatros domínios (Físico, Psicológico, Meio ambiente e Relações sociais), calculando-se também a média populacional para cada domínio.

Para se verificar as relações entre as variáveis estudadas, optou-se pela utilização de testes e análises não-paramétricas, dentre os quais: o teste do quiquadrado, para verificar a variação da proporção entre variáveis categóricas; o teste $U$ de Mann-Whitney, para verificar se há relação entre uma variável intervalar e uma variável categórica dicotômica; e o teste de Kruskal-Wallis, para verificar se uma variável intervalar varia de acordo com uma categórica com mais de duas opções. Em todos os testes utilizou-se o nível de significância de $5 \%$.

\section{RESULTADOS}

A partir das análises descritivas com as variáveis categóricas de cunho sociodemográfico pode-se observar que a amostra desse estudo é composta em sua maior parte por trabalhadores que possuem uma formação complementar, como pós-graduação ou especialização, além de terem um contrato de vínculo efetivo. Entretanto, a remuneração média declarada pelos participantes é de três mil reais, o que não é muito alto para um profissional com tal qualificação. Ademais, é notável o dado obtido de que um quarto dos profissionais terem afirmado já ter se ausentado ou faltado ao trabalho por motivo de doença 
relacionada à atividade que desempenham. Esses e outros dados descritivos a respeito da amostra estão apresentados nas Tabelas 1 e 2, a seguir.

Tabela 1. Variáveis categóricas dicotômicas de características sociodemográficas e do trabalho da amostra.

\begin{tabular}{|c|c|c|}
\hline Variável & Frequência & Porcentagem \\
\hline \multicolumn{3}{|l|}{ Sexo } \\
\hline Feminino & 27 & 84,4 \\
\hline Masculino & 5 & 15,6 \\
\hline \multicolumn{3}{|l|}{ Você possui filhos? } \\
\hline Sim & 11 & 34,4 \\
\hline Não & 21 & 65,6 \\
\hline \multicolumn{3}{|l|}{ Se sim, quantos? } \\
\hline Um & 7 & $63,6 *$ \\
\hline Dois & 3 & $27,3 *$ \\
\hline Três & 1 & $9,1^{*}$ \\
\hline \multicolumn{3}{|c|}{$\begin{array}{l}\text { Possui alguma pós-graduação ou } \\
\text { especialização? }\end{array}$} \\
\hline Sim & 25 & 78,1 \\
\hline Não & 7 & 21,9 \\
\hline \multicolumn{3}{|l|}{ Tipo de vínculo } \\
\hline Concursado/efetivo & 19 & 59,4 \\
\hline Contrato temporário & 13 & 40,6 \\
\hline \multicolumn{3}{|c|}{$\begin{array}{l}\text { Já precisou tirar licença-saúde do } \\
\text { trabalho? }\end{array}$} \\
\hline $\operatorname{Sim}$ & 13 & 40,6 \\
\hline Não & 19 & 59,4 \\
\hline \multicolumn{3}{|c|}{$\begin{array}{l}\text { Já precisou se ausentar ou faltar } \\
\text { por motivo de doença relacionada ao } \\
\text { trabalho? }\end{array}$} \\
\hline Sim & 8 & 25 \\
\hline Não & 24 & 75 \\
\hline
\end{tabular}

Nota. $*=$ Porcentagem válida. 
Tabela 2. Variáveis intervalares de características sociodemográficas e do trabalho da amostra.

\begin{tabular}{|c|c|c|c|c|}
\hline Variável & Média & $\begin{array}{l}\text { Desvio- } \\
\text { padrão }\end{array}$ & Mínimo & Máximo \\
\hline Idade & 35,03 & 9,15 & 24 & 60 \\
\hline Carga horária de trabalho & 35,88 & 6,45 & 20 & 50 \\
\hline Remuneração (em reais) & 3000,42 & 1498,06 & 935,00 & 8000,00 \\
\hline $\begin{array}{l}\text { Tempo no trabalho (em } \\
\text { meses) }\end{array}$ & 39,94 & 41,60 & 1 & 180 \\
\hline
\end{tabular}

Em relação à qualidade de vida dos participantes, observa-se que os escores obtidos (Tabela 3) nas quatro dimensões propostas no instrumento são um pouco abaixo dos valores de referência descritos por Fleck et al. (2000). Assim, é possível afirmar que os psicólogos da amostra percebem um comprometimento na qualidade de suas vidas. No que se refere aos dados obtidos através do $J C Q$, com relação às características do trabalho (Tabela 3), os participantes obtiveram escores ligeiramente elevados na dimensão do "Controle sobre o trabalho", demonstrado que possuem, de certa forma, uma variedade de habilidades a serem usadas e alguma possibilidade de decidir sobre o trabalho que desenvolvem. Já com relação à "Demanda psicológica do trabalho", os trabalhadores demonstraram ter altas exigências psicológicas nas tarefas que realizam. Desse modo, a partir do modelo proposto por Karasek (1979), o trabalho desses profissionais se configura enquanto um "Trabalho ativo".

Tabela 3.

Qualidade de vida e características do trabalho da amostra.

\begin{tabular}{lll}
\hline Variável & Média & Desvio-padrão \\
\hline WHOQOL-bref & & \\
$\quad$ Domínio físico & 13,84 & 2,72 \\
$\quad$ Domínio psicológico & 13,52 & 2,8 \\
$\quad$ Meio ambiente & 13,6 & 3,32 \\
$\quad$ Relações sociais & 12,28 & 2,6 \\
JCQ & & \\
Controle sobre o trabalho & 70,94 & 6,12 \\
$\quad$ Uso de habilidade & 35,31 & 4,65 \\
$\quad$ Autoridade decisória & 35,63 & 5,41 \\
Demanda psicológica do trabalho & 35,47 & 8,64 \\
$\quad$ Insegurança no trabalho & 6,50 & 3,26 \\
$\quad$ Apoio social & 22,88 & 8,39 \\
\hline
\end{tabular}

Por meio do cálculo dos escores do $S R Q-20$, verificou-se que houve uma variação de zero a 19 respostas positivas, com metade da população, ou seja, 16 
participantes respondendo positivamente a sete ou menos questões, e a outra metade com pontuação acima do ponto de corte. Com isso, obteve-se então que em metade da população há uma probabilidade de presença de transtorno nãopsicótico, ou seja, distúrbios psíquicos menores. Os participantes foram categorizados, então, por meio da variável dicotômica $d p m$, e fez-se o cruzamento com as demais variáveis dicotômicas, por meio do teste quiquadrado, com o intuito de verificar se a distribuição dos participantes com escores a partir de oito está associada com algum dos demais grupos da amostra. Observou-se que a categoria "Já precisou se ausentar ou faltar por motivo de doença relacionada ao trabalho" apresentou uma relação significativa $(p=, 037 ; F E T)$, recusando-se, assim, a hipótese nula de que não há associação entre esses grupos. Também se observou uma relação significativa com a variável referente ao nível de demanda psicológica do trabalho $(p=, 006 ; F E T)-$ Tabela 4.

Tabela 4. Prevalência de distúrbios psíquicos menores (dpm) segundo níveis de demanda psicológica, controle sobre o próprio trabalho e apoio social no trabalho.

\begin{tabular}{llll}
\hline Características & $\mathrm{n}$ & $d p m$ & $p$ valor \\
\hline $\begin{array}{l}\text { Demanda Psicológica } \\
\quad \text { Baixa }\end{array}$ & 10 & 1 & \\
$\quad$ Alta & 22 & 15 & 1,000 (FET) \\
$\begin{array}{l}\text { Controle } \\
\quad \text { Baixo }\end{array}$ & 1 & 1 & \\
$\quad$ Alto & 31 & 15 &, 006 (FET) \\
Apoio Social & & & \\
$\quad$ Baixo & 16 & 10 &, 157 \\
$\quad$ Alto & 16 & 6 & \\
\hline
\end{tabular}

Com a finalidade de se verificar a existência de diferenças estatisticamente significativas entre a provável presença de transtornos mentais não-psicóticos (SRQ-20) e a qualidade de vida (WHOQOL-bref) e as características do trabalho (JCQ), realizou-se o teste $U$ de Mann-Whitney (Tabela 5). Por meio desse teste foi possível observar uma relação significativa entre a variável $d p m$ e as quatro dimensões do WHOQOL-bref, sendo que os participantes com dpm obtiveram escores menores nas quatro dimensões, ou seja, apresentavam uma qualidade de vida inferior aos participantes sem $d p m$. Além disso, a variável $d p m$ também apresentou relação significativa com a dimensão "Demanda psicológica do trabalho", mostrando que participantes com $d p m$ tiveram escores mais altos com relação à demanda psicológica que percebem na realização do seu trabalho. No entanto, não se constatou diferenças significativas com as demais dimensões do $J C Q$. 
Tabela 5. Relação entre variáveis a partir do Teste U de Mann-Whitney.

\begin{tabular}{|c|c|c|}
\hline Variáveis & $\mathrm{U}$ & $p$ valor \\
\hline \multicolumn{3}{|l|}{$d p m$} \\
\hline Domínio físico & 24,500 & 000 \\
\hline Domínio psicológico & 11,000 & 000 \\
\hline Relações sociais & 33,500 & 000 \\
\hline Meio ambiente & 28,500 & 000 \\
\hline Demanda psicológica do trabalho & 65,000 & ,017 \\
\hline \multicolumn{3}{|l|}{ Sexo } \\
\hline Domínio físico & 31,500 & , 061 \\
\hline Demanda psicológica & 29,500 & ,048 \\
\hline \multicolumn{3}{|l|}{ Possui filhos } \\
\hline Relações sociais & 67,000 & ,051 \\
\hline \multicolumn{3}{|l|}{ Possui pós } \\
\hline Controle sobre o trabalho & 30,500 & ,009 \\
\hline \multicolumn{3}{|l|}{ Tipo de vínculo } \\
\hline Meio ambiente & 73,500 & , 054 \\
\hline Insegurança no trabalho & 14,500 & 000 \\
\hline \multicolumn{3}{|l|}{ Já tirou licença- saúde do trabalho } \\
\hline Domínio físico & 59,000 & ,013 \\
\hline Domínio psicológico & 74,500 & ,059 \\
\hline Apoio social & 73,500 & 052 \\
\hline
\end{tabular}

Já precisou se ausentar ou faltar por algum motivo de doença relacionado à função que desempenha

Domínio físico

$21,000,001$

Dominío psicológico

$51,500,051$

Relações sociais

$35,000,007$

Controle sobre o trabalho

$49,000,039$

Demanda psicológica

$17,000,001$

Nível de demanda psicológica

Domínio físico

$51,000,016$

Domínio psicológico

$50,000,014$

Meio ambiente

$57,500,032$

Também foram testadas as relações entre as dimensões das escalas de qualidade de vida e de características do trabalho com as demais categorias dicotômicas (sexo, se possui filhos, se possui pós, tipo de vínculo, se já tirou 
licença-saúde, e se já precisou se ausentar ou faltar) por meio do teste $U$ de Mann-Whitney. Através desses cruzamentos, pôde-se notar que a variável Sexo se diferenciou significativamente da dimensão "Demanda psicológica de trabalho", com participantes do sexo feminino apresentando escores mais elevados com relação a essa característica do trabalho. Além disso, essa variável apresentou um valor marginal com relação à dimensão "Domínio físico", com mulheres apresentando uma pior qualidade de vida nesse domínio. A variável "Possui filhos" não se relacionou significativamente com nenhuma das dimensões. Contudo, apresentou um valor de $p=, 051$ frente a dimensão "Relações sociais", demonstrando que o fato dos participantes possuírem filhos diminui a qualidade de vida percebida nas relações sociais.

A variável "Possui pós", referente ao nível de formação do participante, diferiu significativamente apenas com relação à dimensão "Controle sobre o trabalho", com profissionais que possuem algum tipo de formação complementar apresentando um maior controle sobre o trabalho que desempenham. Já o "Tipo de vínculo" do participante, apresentou uma relação significativa com a dimensão "Insegurança no trabalho", sendo que os profissionais de vínculo temporário se sentem mais inseguros no seu trabalho do que os com vínculo efetivo/concursado. Além de apresentar um valor de $\mathrm{p}$ marginal frente à dimensão "Meio ambiente", com $p=, 054$, com os profissionais efetivos apresentando uma melhor qualidade de vida nessa dimensão. A categoria "Já tirou licença-saúde do trabalho" apresentou relação significativa com o "Domínio físico", além de valores marginais de p com relação ao "Domínio psicológico" $(p=, 059)$ e ao "Apoio social" $(p=, 052)$, sendo que os profissionais que tiraram licença pontuaram mais baixo nos domínios da qualidade de vida e mais alto com relação ao apoio social que percebem no ambiente de trabalho.

Por fim, a variável "Já precisou se ausentar ou faltar por algum motivo de doença relacionado à função que desempenha" diferiu significativamente com relação às dimensões "Domínio físico" e "Relações sociais", e apresentou um valor muito próximo diante do "Domínio psicológico" $(p=, 051)$, com os profissionais que disseram já ter se ausentado exibindo uma pior qualidade de vida nesses três domínios. Com relação ao $J C Q$, essa variável apresentou relação significativa com as dimensões "Controle sobre o trabalho" e "Demanda psicológica do trabalho", em que os profissionais que já se ausentaram perceberam um maior controle sobre o trabalho, mas também uma maior demanda psicológica.

Além disso, buscou-se verificar também as relações entre as características do trabalho com a qualidade de vida dos participantes, através do teste $U$ de Mann-Whitney. Para isso, cruzou-se os indicadores dicotomizados (alto e baixo) do JCQ com as quatro dimensões do WHOQOL-bref. Pôde-se observar que o indicador "Nível de demanda psicológica" diferiu significativamente do "Domínio físico", do "Domínio psicológico" e da dimensão 
"Meio ambiente", sendo que os participantes que relataram um nível elevado de demanda psicológica apresentaram escores baixos nas dimensões de qualidade de vida. Os demais indicadores não apresentaram valores de $p$ significativos na relação com as dimensões da qualidade de vida.

\section{DISCUSSÃO}

As características do trabalho observadas a partir do modelo demandacontrole neste estudo, configuram-se no quadrante de trabalho ativo, que, teoricamente, não seria uma situação de trabalho adoecedora. No entanto, houve um elevado índice de distúrbios psíquicos $(d p m)$, mesmo em uma situação de trabalho com alta demanda e alto controle corroborando os achados de outros estudos (Araújo, Aquino et al., 2003; Araújo, Graça et al., 2003; Nascimento Sobrinho et al., 2006). Segundo os autores, isso pode demonstrar que a demanda psicológica tem relevância maior do que o controle sobre a atividade, para a prevalência de $d p m$. Na presente pesquisa também foi possível observar que a dimensão demanda psicológica apresentou relação negativa com a qualidade de vida da amostra, ou seja, a elevada exigência psicológica se associou com uma qualidade de vida prejudicada. No entanto, não se verificou a mesma relação quanto ao controle.

O adoecimento mental dos trabalhadores nessa configuração de trabalho também pode ser explicado pelo fato de que a dimensão controle é composta pelos fatores autoridade decisória e uso de habilidades (Karasek, 1979), em que o segundo fator pode contribuir para uma situação de maior adoecimento no trabalho, por tratar de questões como a diversidade de tarefas a serem desenvolvidas, bem como a repetitividade e a criatividade exigidas na realização do trabalho, e a autonomia, que pode ser encarada como um aumento de responsabilidade e de pressão (Araújo, Graça et al., 2003). Ora, a atuação do psicólogo na Atenção Primária envolve um número altíssimo de atividades a serem realizadas, de acordo com as demandas locais e o modo de atuação de cada profissional, conforme pode ser observado no relatório do Conselho Federal de Psicologia (2010).

Dessa forma, a quantidade de atividades no trabalho parece contribuir para o adoecimento dos trabalhadores, podendo estar associado a uma elevada carga de trabalho (Araújo, Graça et al., 2003). Além disso, ressalta-se a existência de "um hiato entre a formação encontrada nos cursos de Psicologia e as demandas dessa inserção" (Romagnoli, 2009, p.527), fazendo com que os profissionais adentrem os espaços de trabalho e venham a desempenhar suas funções, sem muita clareza do que e de como realizarem seus afazeres, ou seja, sem muitas possibilidades de ação frente às exigências do trabalho.

Nesse sentido, pode-se questionar a relação entre a dimensão de controle relatada pelos psicólogos e um modo de trabalho ativo e não adoecedor, assim 
como apontado nos estudos de Araújo, Aquino et al. (2003) e Araújo, Graça et al. (2003). Diante da realidade de trabalho encontrada por esses profissionais no desenvolver de suas atividades, o controle relatado pode ser entendido como uma grande autonomia que, por sua vez, pode se traduzir em um aumento das responsabilidades e da carga de trabalho desses profissionais. Dessa forma, os efeitos positivos do controle aparentam estar reduzidos frente aos efeitos negativos da alta demanda psicológica.

Falzon e Sauvagnac (2007) destacam que há um vínculo entre a carga de trabalho e os modos operatórios, onde a depender da carga de trabalho e do nível de exigência da tarefa, o trabalhador venha a adotar um ou outro modo operatório. Por outro lado, um modo operatório adotado pelo trabalhador faz com que a carga de trabalho aumente ou diminua. No caso dos psicólogos da APS, observa-se que a ausência de um repertório mais amplo de modos operatórios, que poderiam ter obtido na formação, contribui para um aumento da carga de trabalho. Então eles se encontram em uma situação de alta demanda psicológica e, por mais que aparentem ter controle sobre o trabalho, acabam adoecendo no desenvolvimento de suas atividades, devido às exigências que encontram sem dispor de modos operatórios mais apropriados para lidar com elas.

Com relação à qualidade de vida, foi possível observar, como dito anteriormente, que os profissionais participantes desse estudo apresentaram uma baixa pontuação nas quatro dimensões propostas pelo Whoqol-bref. Fogaça, Carvalho, Nogueira e Martins (2009) afirmam que a associação entre adoecimento mental no trabalho e qualidade de vida tem sido avaliada em diversos trabalhos, mostrando um grau de comprometimento da qualidade de vida física e psicológica em situações de alta demanda psicológica no trabalho. Nossos achados corroboram essa literatura, apontando um impacto significativo da demanda psicológica do trabalho nas dimensões física, psicológica e meio ambiente da qualidade de vida. Desse modo, os psicólogos, além de apresentarem uma alta prevalência de $d p m$, apresentam uma baixa qualidade de vida, devido às características do trabalho a que estão submetidos.

Fogaça et al. (2009), afirmam ainda que estudos demonstram relações entre a alta demanda psicológica e o estresse no trabalho, sendo que mulheres apresentam maior prevalência de depressão e estresse no trabalho. Com isso, os autores buscam indicar uma possível relação entre gênero e adoecimento no trabalho. No presente estudo, observou-se uma relação significativa entre sexo e demanda psicológica do trabalho, além de uma possível relação com o domínio físico da qualidade de vida. Apesar de não ter sido verificada uma relação significativa entre $d p m$ e a variável sexo, supõe-se que as mulheres podem ter maior prevalência de adoecimento mental no trabalho, pelo fato de serem mais afetadas pelas altas demandas psicológicas do trabalho, que se associa a nível 
mais altos de estresse e depressão (Araújo, Godinho, Reis, \& Almeida, 2006; Fogaça et al., 2009).

Um fato relevante que foi possível observar durante o processo de coleta e de divulgação desta pesquisa é a baixa participação dos profissionais da categoria nos espaços e nas reuniões organizados tanto pelo Conselho de Classe como pelo Sindicato. Por exemplo, apenas três participantes responderam que são membros do Sindicato de profissionais (questão 14 do JCQ). Além disso, nas reuniões em que o pesquisador esteve, o número de presentes não era superior a dez, incluindo os responsáveis pela condução da reunião. Esses espaços coletivos apresentam-se como possibilidade para discussão, reflexão, construção e apoio, frente as exigências encontradas nas situações de trabalho, propiciando aos trabalhadores alternativas para lidar com a alta demanda psicológica e o adoecimento mental.

\section{CONSIDERAÇÕES FINAIS}

É importante esclarecer aqui que inicialmente esperava-se um número maior de respostas ao questionário, sendo desejável uma amostra de no mínimo 100 respostas, afim de satisfazer a recomendação do Job Content Questionnaire User's Guide (Karasek, 1985). Tal número possibilitaria a realização de análises estatísticas mais apuradas e com maior grau de confiabilidade. No entanto, ao longo do primeiro mês da coleta observou-se que a quantidade de respostas estava muito abaixo, e a expectativa passou a ser, então, de uma amostra com no mínimo 50 respostas, o que diminuiria a qualidade das análises, mas mantendo um bom grau de confiabilidade.

Diversas estratégias foram utilizadas a fim de viabilizar um aumento do número de respostas, além dos procedimentos inicialmente definidos. A divulgação do questionário por meio da articulação com o sindicato da categoria, com o fórum de trabalhadores, além do compartilhamento do link do questionário em páginas e grupos de psicólogos de redes sociais, lista de e-mails de ex-alunos dos cursos de graduação e pós-graduações em Psicologia da UFES, foram alguns dos modos encontrados para ampliar o número de respostas. Porém, não houve um aumento significativo nesse número, e ao final do período pré-estabelecido para a disponibilização do questionário online, apenas 32 respostas foram registradas.

Assim, entende-se a fragilidade das análises realizadas e sugere-se a realização de estudos posteriores com um número maior de participantes nessa configuração de trabalho. A partir dos dados obtidos, indicando baixos níveis de qualidade de vida, o elevado índice de adoecimento mental e a configuração de trabalho ativo, no entanto, de muita variabilidade, conclui-se que a situação de trabalho dos psicólogos na APS é de agressão à saúde. Faz-se necessário buscar estratégias para melhorar as condições de trabalho e possibilitar ao trabalhador 
o desenvolvimento de suas atividades de maneira mais saudável. Indicam-se que tais estratégias devam focar em: diminuição dos níveis de demanda psicológica do trabalho, diminuindo a pressão do tempo e a interrupção das tarefas; descrição mais clara a respeito das atividades a serem realizadas nesses espaços, possibilitando um maior controle sobre as tarefas; ampliação do repertório de modos operatórios dos profissionais, estabelecendo novas formas de lidar com as exigências do trabalho por meio da formação; aumento da participação de profissionais em espaços coletivos que possibilitem uma construção coletiva de conhecimentos sobre as tarefas a serem realizadas.

\section{REFERÊNCIAS}

Araújo, T. M., Aquino, E., Menezes, G., Santos, C. O., \& Aguiar, L. (2003). Aspectos psicossociais do trabalho e distúrbios psíquicos entre trabalhadoras de enfermagem. Revista de Saúde Pública, 37(4), 424-433. doi: 10.1590/S0034-89102003000400006

Araújo, T. M., Godinho, T. M., Reis, E. J. F. B., \& Almeida, M. M. G. (2006). Diferenciais de gênero no trabalho docente e repercussões sobre a saúde. Ciência \& Saúde Coletiva, 11(4), 1117-1129. doi:10.1590/S141381232006000400032

Araújo, T. M., Graça, C. C., \& Araújo, E. (2003). Estresse ocupacional e saúde: contribuições do Modelo Demanda-Controle. Ciência \& Saúde Coletiva, 8(4), 991-1003. doi:10.1590/S1413-81232003000400021

Araújo, T. M., \& Karasek, R. (2008). Validity and reliability of the job content questionnaire in formal and informal jobs in Brazil. Scandinavian Journal of Work, Environment \& Health Supplements, (6), 52-59.

Araújo, T. M., Sena, I. P., Viana, M. A., \& Araújo, E. M. (2005). Mal-estar docente: avaliação de condições de trabalho e saúde em uma instituição de ensino superior. Revista Baiana de Saúde Pública, 29(1), 6-21.

Archanjo, A. M., \& Schraiber, L. B. (2012). A atuação dos psicólogos em unidades básicas de saúde na cidade de São Paulo. Saúde e Sociedade, 21(2), 351-363. doi:10.1590/S0104-12902012000200009

Boarini, M. L., \& Borges, R. F. (2009). O psicólogo na atenção básica à saúde. Psicologia: Ciência e Profissão, 29(3), 602-613. doi:10.1590/S141498932009000300013

Borges, L. de O., \& Argolo, J. C. T. (2002). Adaptação e validação de uma escala de bem-estar psicológico para uso em estudos ocupacionais. Avaliação Psicológica, 1(1), 17-27.

Borsoi, I. C. F. (2007). Da relação entre trabalho e saúde à relação entre trabalho e saúde mental. Psicologia \& Sociedade, 19(1), 103-111. doi: $10.1590 /$ S0102-71822007000400014 
Brotto, T. C. A., \& Dalbello-Araujo, M. (2012). É inerente ao trabalho em saúde o adoecimento de seu trabalhador?. Revista Brasileira de Saúde Ocupacional, 37(126), 290-305. doi:10.1590/S0303-76572012000200011

Cardoso, J. P., Ribeiro, I. de Q. B., Araújo, T. M. de, Carvalho, F. M., \& Reis, E. J. F. B. dos. (2009). Prevalência de dor musculoesquelética em professores. Revista Brasileira de Epidemiologia, 12(4), 604-614. doi:10.1590/S1415790X2009000400010

Clot, Y. (2007). A função psicológica do trabalho. Petrópolis, RJ: Vozes.

Clot, Y. (2010). Trabalho e poder de agir. Belo Horizonte, MG: Fabrefactum.

Conselho Federal de Psicologia. (2010). Práticas profissionais de psicólogos e psicólogas na atenção básica à saúde. Brasília, DF: CFP/CREPOP.

De Marco, P. F., Cítero, V. de A., Moraes, E., \& Nogueira-Martins, L. A. (2008). O impacto do trabalho em saúde mental: transtornos psiquiátricos menores, qualidade de vida e satisfação profissional. Jornal Brasileiro de Psiquiatria, 57(3), 178-183. doi: 10.1590/S0047-20852008000300004

Dimenstein, M. D. B. (1998). O psicólogo nas Unidades Básicas de Saúde: desafios para a formação e atuação profissionais. Estudos de Psicologia (Natal), 3(1), 53-81. doi:10.1590/S1413-294X1998000100004

Doppler, F. (2007). Trabalho e saúde. In P. Falzon (Ed.), Ergonomia (pp. 47-59). São Paulo, SP: Editora Blücher Ltda, 2007.

Elias, M. A., \& Navarro, V. L. (2006). A relação entre o trabalho, a saúde e as condições de vida: negatividade e positividade no trabalho das profissionais de enfermagem de um hospital escola. Revista Latino-Americana de Enfermagem, 14(4), 517-525. doi:10.1590/S0104-11692006000400008.

Falzon, P., \& Sauvagnac, C. (2007). Carga de trabalho estresse. In P. Falzon (Ed.), Ergonomia (pp. 141-154). São Paulo, SP: Editora Blücher Ltda, 2007.

Filgueiras, J. C., \& Hilppert, M. I. (2002). Estresse: Possibilidades e limites. In M. da G. Jacques, \& W. Codo (Eds.), Saúde mental e trabalho: Leituras (pp. 112-129). Petrópolis, RJ: Vozes.

Fleck, M. P. de A., Leal, O. F., Louzada, S., Xavier, M., Chachamovich, E., ..., \& Pinzon, V. (1999). Desenvolvimento da versão em português do instrumento de avaliação de qualidade de vida da OMS (WHOQOL-100). Revista Brasileira de Psiquiatria, 21(1), 19-28. doi:10.1590/S151644461999000100006

Fleck, M. P. de A., Louzada, S., Xavier, M., Chachamovich, E., Vieira, G., ..., \& Pinzon, V. (2000). Aplicação da versão em português do instrumento abreviado de avaliação da qualidade de vida "WHOQOL-bref". Revista de Saúde Pública, 34(2), 178-183. doi:10.1590/S0034-89102000000200012 
Fogaça, M. de C., Carvalho, W. B. de, Nogueira, P. C. K., \& Martins, L. A. N. (2009). Estresse ocupacional e suas repercussões na qualidade de vida de médicos e enfermeiros intensivistas pediátricos e neonatais. Revista Brasileira de Terapia Intensiva, 21(3), 299-305. doi:10.1590/S0103$507 \times 2009000300010$

Freitas, C. P. P. (2013). Avaliação de impacto de uma tecnologia social para profissionais de psicologia que trabalham com vítimas de violência sexual. Dissertação de Mestrado, Instituto de Psicologia, Universidade Federal do Rio Grande do Sul, Porto Alegre.

Gonçalves, D. M., Stein, A. T., \& Kapczinski, F. (2008). Avaliação de desempenho do Self-Reporting Questionnaire como instrumento de rastreamento psiquiátrico: um estudo comparativo com o Structured Clinical Interview for DSM-IV-TR. Cadernos de Saúde Pública, 24(2), 380-390. doi:10.1590/S0102-311X2008000200017

Greco, P., Magnago, T., Prochnow, A., Beck, C., \& Tavares, J. (2011). Utilização do modelo demanda-controle de Karasek na América Latina: uma pesquisa demográfica. Revista de Enfermagem da UFSM, 1(2), 272-281.

Guérin, F., Laville, A., Daniellou, F., Duraffourg, J., \& Kerguelen, A. (2001). Compreender o trabalho para transformá-lo: a prática da ergonomia. São Paulo, SP: Edgard Blücher.

Jacques, M. G. C. (2003). Abordagens teórico-metodológicas em saúde/doença mental \& trabalho. Psicologia \& Sociedade, 15(1), 97-116. doi: $10.1590 /$ S0102-71822003000100006

Karasek, R. A. (1979). Job demands, job decision latitude, and mental strain: implications for job redesign. Administrative Science Quarterly, 24(2), 285308. doi: $10.2307 / 2392498$

Karasek, R. A. (1985). Job Content Questionnaire and User's Guide. Lowell, MA: University of Massachusetts.

Kirchhof, A. L. C., Magnago, T. S. B. de S., Camponogara, S., Griep, R. H., Tavares, J. P., Prestes, F. C., \& Paes, L. G.. (2009). Condições de trabalho e características sócio-demográficas relacionadas à presença de distúrbios psíquicos menores em trabalhadores de enfermagem. Texto \& Contexto Enfermagem, 18(2), 215-223. doi:10.1590/S0104-07072009000200003

Knuth, B. S., Silva, R. A. da, Oses, J. P., Radtke, V. A., Cocco, R. A., \& Jansen, K. (2015). Mental disorders among health workers in Brazil. Ciência \& Saúde Coletiva, 20(8), 2481-2488. doi:10.1590/141381232015208.05062014

Lacaz, F. A. de C. (2000). Qualidade de vida no trabalho e saúde/doença. Ciência \& Saúde Coletiva, 5(1), 151-161. doi:10.1590/S1413-81232000000100013 
Mari, J. J., \& Williams, P. (1985). A comparison of the validity of two psychiatric screening questionnaires (GHQ-12 and SRQ-20) in Brazil, using relative operating characteristic (ROC) analysis. Psychological Medicine, 15(3), 651659. doi: $10.1017 /$ S0033291700031500

Mari, J. J., \& Williams, P. (1986). A validity study of a psychiatric screening questionnaire (SRQ-20) in primary care in the city of São Paulo. The British Journal of Psychiatry, 148(1), 23-26. doi:10.1192/bjp.148.1.23

Martinez, M. C. (2002). As relações entre a satisfação com aspectos psicossociais no trabalho e a saúde do trabalhador. Dissertação de Mestrado, Faculdade de Saúde Pública, Universidade de São Paulo.

Martinez, M. C., Paraguay, A. I. B. B., \& Latorre, M. do R. D. de O. (2004). Relação entre satisfação com aspectos psicossociais e saúde dos trabalhadores. Revista de Saúde Pública, 38(1), 55-61. doi:10.1590/S003489102004000100008

Marx, K. (2008). O processo de trabalho ou o processo de produzir valores-deuso. In K. Marx, O Capital: crítica da economia política (26. ed., pp. 211219). Rio de Janeiro, RJ: Civilização Brasileira.

Medeiros, M. A. V. de, Nunes, M. L. T., \& Melo, F. da F. M. de. (2012). Saúde mental de psicólogos trabalhadores na saúde pública: um estudo a partir de Dejours e Freud. Anais do VIII Seminário do Trabalho: Trabalho e Políticas Sociais no Século XXI. Marília, SP: UNESP.

Mendes, R. (1988). Impacto dos efeitos da ocupação sobre a saúde dos trabalhadores. I. Morbidade. Revista de Saúde Pública, 22(4), 311-326. doi: $10.1590 /$ S0034-89101988000400007

Minayo, M. C. de S., Hartz, Z. M. de A., \& Buss, P. M. (2000). Qualidade de vida e saúde: um debate necessário. Ciência \& Saúde Coletiva, 5(1), 7-18. doi: $10.1590 /$ S1413-81232000000100002

Nascimento Sobrinho, C. L., Carvalho, F. M., Bonfim, T. A. S., Cirino, C. A. S., \& Ferreira, I. S. (2006). Condições de trabalho e saúde mental dos médicos de Salvador, Bahia, Brasil. Cadernos de Saúde Pública, 22(1), 131-140. doi: 10.1590/S0102-311X2006000100014

Nassif, L. F., A. (2005). Origens e desenvolvimento da Psicopatologia do Trabalho na França (século XX): uma abordagem histórica. Memorandum, $8,79-87$.

Rocha, V. M. da, \& Fernandes, M. H. (2008). Qualidade de vida de professores do ensino fundamental: uma perspectiva para a promoção da saúde do trabalhador. Jornal Brasileiro de Psiquiatria, 57(1), 23-27. doi: 10.1590/S0047-20852008000100005

Romagnoli, R. C. (2009). Breve estudo institucionalista acerca do Programa de Saúde da Família. Saúde e Sociedade, 18, 525-536. doi:10.1590/S010412902009000300016 
Sanna, M. C. (2007). Os processos de trabalho em Enfermagem. Revista Brasileira de Enfermagem, 60(2), 221-224. doi:10.1590/S003471672007000200018

Santos, K. O. B. (2006). Estresse ocupacional e saúde mental: desempenho dos instrumentos de avaliação em populações de trabalhadores na Bahia, Brasil. Dissertação de Mestrado, Programa de Pós-graduação em Saúde Coletiva, Universidade Estadual de Feira de Santana, Feira de Santana.

Seligmann-Silva, E. (1994). Desgaste mental no trabalho dominado. Rio de Janeiro, RJ: Editora UFRJ/ Editora Cortez.

Silva, A. B. H. C. d. (2010). O estresse na prática profissional do psicólogo em UTI: uma revisão de literatura. Revista da SBPH, 13(1), 33-51.

Sobre os autores

Lucas Có Barros Duarte é psicólogo pela Universidade Federal do Espírito Santo e mestre pelo Programa de Pós-Graduação em Psicologia da Universidade Federal do Espírito Santo. Trabalha na linha Psicologia e Saúde e recebeu financiamento de pesquisa da CAPES. lucascobarrosduarte@gmail.com

Thiago Drumond Moraes é psicólogo pela Universidade Federal do Espírito Santo, mestre em psicologia pela Universidade Federal Fluminense e doutor em psicologia social pela Universidade do Estado do Rio de Janeiro. Trabalha na linha de Psicologia do Trabalho e das Organizações. thiago.moraes@ufes.br

Recebido em: 06/04/2016

Revisado em: 23/09/2016

Aceito em: 01/10/2016 